\title{
Incorporating Proactivity to Context-Aware Recommender Systems for E-Learning
}

\author{
Daniel Gallego, Enrique Barra, Pedro Rodríguez, Gabriel Huecas \\ Departamento de Ingeniería de Sistemas Telemáticos, Universidad Politécnica de Madrid \\ Avda. Complutense 30, Ciudad Universitaria, \\ 28040 Madrid, Spain \\ \{dgallego, ebarra, prodriguez, gabriel $\} @$ dit.upm.es
}

\begin{abstract}
Recommender systems in e-learning have proved to be powerful tools to find suitable educational material during the learning experience. But traditional user request-response patterns are still being used to generate these recommendations. By including contextual information derived from the use of ubiquitous learning environments, the possibility of incorporating proactivity to the recommendation process has arisen. In this paper we describe methods to push proactive recommendations to e-learning systems users when the situation is appropriate without being needed their explicit request. As a result, interesting learning objects can be recommended attending to the user's needs in every situation. The impact of this proactive recommendations generated have been evaluated among teachers and scientists in a real e-learning social network called Virtual Science Hub related to the GLOBAL excursion European project. Outcomes indicate that the methods proposed are valid to generate such kind of recommendations in e-learning scenarios. The results also show that the users' perceived appropriateness of having proactive recommendations is high.
\end{abstract}

Keywords-Proactivity, Context-awareness, E-learning, Recommender Systems

\section{INTRODUCTION}

Recommender systems have proved to be powerful information filtering tools that help users to find content, products or services adapted to their needs. In the last years their application in the e-learning domain has become an important research field [1]. More recently, the incorporation of contextual information about the users and their environment has attracted major interest [2] as these context-aware recommender systems allow to generate more accurate and personalized recommendations. As a consequence of knowing this contextual information, innovative recommendation techniques can be studied to improve the traditional user request-response pattern usually presented in almost every recommender system.

Following the model we proposed in [3], in this paper we present the methods needed to incorporate proactivity in a context-aware recommender system for e-learning platforms. In addition, we evaluate the appropriateness of proactivity when recommending learning content to educators in order to help them creating better educational material for their daily classes attending to their students' necessities.

The methods proposed have been applied in a real scenario: the Virtual Science $\mathrm{Hub}^{1}(\mathrm{ViSH})$, a social e-learning network related to the GLOBAL excursion ${ }^{2}$ (Extended Curriculum for Science Infrastructure Online) European project. The system developed is able to generate proactive recommendations of learning objects (LOs) to teachers and scientists in order to help them to create the lessons that their students will consume.

Regarding the impact of proactivity for educators, an evaluation among 104 users (i.e. teachers and scientists) has been performed to generate a user model related to the appropriateness of proactive recommendations. This study covers not only the applicability of the methods proposed to a real deployed platform, but also the impact of proactivity in the user experience related to the educational activity of teachers and scientists collaborating in ViSH. The results obtained have led us to build user interfaces that nowadays are providing proactive recommendations to ViSH users.

The article is organized as follows: first, we present related work that positions this work within existing research that has been conducted in the area of recommender systems for e-learning. Then, the ViSH scenario is described. After that, we detailed the methods used to assess proactivity. In the following section the results from the evaluations carried out are presented. Section VI illustrates the proactive user interfaces implemented in ViSH as a result of the outcomes achieved. Finally, some concluding remarks and future work are outlined.

\section{RELATED WORK}

\section{A. Recommender systems in e-learning}

Attending to the survey of recommender systems in Technology Enhanced Learning (TEL) presented by Manouselis et al. [1] the main feature these systems offer consists of recommending learning resources [4]; but people [5] and activities [6] that may be important in the learning experience are also suggested in many of them. These functionalities are usually applied in TEL environments like learning networks [7] and teaching communities [8], as well as personal learning environments [9].

According to [1], in TEL a careful analysis of the targeted users and their supported tasks should be carried out. A

\footnotetext{
${ }^{1}$ http://vishub.org

${ }^{2} \mathrm{http} / / / \mathrm{www}$.globalexcursion-project.eu
} 
great number of user attributes, domain characteristics, and intelligent methods can be engaged to provide personalized recommendations. Every e-learning system has its own particularities, but Manouselis et al. [1] highlight some that are quite common in these systems and that have to be considered when designing and implementing a recommender system for TEL.

However, additional context dimensions can be incorporated to improve the level of personalization and accuracy of the recommendations.

\section{B. Context and Proactivity}

Several definitions of context can be found, but we follow one of the most cited definitions proposed by Dey et al. [10] where: "Context is any information that can be used to characterize the situation of an entity. An entity is a person, place, or object that is considered relevant to the interaction between a user and an application, including the user and applications themselves." Systems that use this contextawareness information to provide enhanced recommendations are called context-aware recommender systems (CARS) [11].

In the learning domain, the application of these systems was recently surveyed by Verbert et al. in [2] wherein a context framework for TEL is also proposed containing several dimensions that are relevant for them (e.g. location, time, physical conditions or activity).

One specific research line that is getting very popular is the introduction of proactivity in CARS. These systems push recommendations to the user when the current situation seems appropriate, without explicit user request [12], going beyond traditional recommender systems.

Nonetheless, in the e-learning domain proactivity has not gained much attention yet and only few researches exist. RuizIniesta et al. [13] propose a proactive recommender system in computer-supported learning that works on repositories of LOs and adapts to the student's profile. The system recommends LOs to the student who can enter a conversational process to refine the proposal. However, this approach is tailored for students and for that reason the requirements of their scenario were quite different from ours, focused on helping teachers to create their lessons in collaboration with scientists.

\section{SCENARIO: THE ViRTUAL SCIENCE HUb}

The demand for a qualified workforce with science, technology, engineering and mathematics (STEM) related skills has increased and will increase even more in the next years. Greater efforts must now be made to highlight STEM as a priority area of education and increase engagement at all levels. So European Commission (EC) has defined the advancement of STEM related skills as one of the priorities for the period 2014-2020 [14].

EC shows its concern about the learning scenario in several reports. [15] points out that teachers face rapidly changing demands, technology is rapidly changing the way people teach and learn, and teachers need support to introduce Information and Communication Technologies (ICT) in their daily work.
The EC Benchmarking Reports [16] identified ICT use in almost one hundred per cent of European schools, but schools are not usually making the most of them.

Taking all this into account, and considering that $\mathrm{e}$ Infrastructures are recognized by the $\mathrm{EC}$ as key to a knowledge-based economy and social cohesion, and so they must have a place in education and training, the GLOBAL excursion project was proposed and approved. Together with end-users, GLOBAL excursion is developing a common understanding, teaching use cases, as well as pedagogical and technical artifacts. The aim of this project is to provide young citizens and their educators (e.g. teachers) across Europe with a range of e-Infrastructures and access to expert knowledge on its usage for a joyful exploration of e-Science through eInfrastructures.

The platform where all GLOBAL excursion activities take place is called Virtual Science Hub. It is open source and has been completely developed by the project members following a participatory design process [17].

\section{SITUATION ASSESSMENT FOR PROACTIVE RECOMMENDATIONS: METHODS}

In [3] we propose a general model for generating proactive recommendations in CARS to be used in e-learning systems. In this paper, we focus our attention in the second phase of that model that is in charge of evaluating several context dimensions to determine whether or not the current situation warrants a proactive recommendation. The most influential context dimensions involved in determining proactivity are:

- Location context: composed by the geographical and temporal contextual information.

- User context: composed by the device and activity contextual information.

\section{A. Determination of appropriateness}

One central question related to proactivity is to determine if a recommendation would be appropriate for a given user context. Attending to the model we follow [3], the system has to calculate a decision score $S 1$ that will be evaluated against a threshold $T 1$. Only if $S 1>T 1$ the recommendation will be triggered.

$T 1$ has to be predetermined or learned empirically after putting into operation the recommender engine because it is domain-dependent. However, for the score $S 1$ we have designed a general method to calculate it so as to be usable as a basis for describing the appropriateness of a situation.

As we mentioned above, the location and user context have several features that have to be treated differently among them. This leads to the introduction of two important properties for those components: Each feature value has an appropriateness factor and each feature has a weight. The first one indicates how appropriate a recommendation would be for this feature value, under the assumption that for all other features, a recommendation would be appropriate. The weight of a feature represents the importance it should have on the decision of 
appropriateness. In the following, formal definitions will be given based on these ideas.

Definition 1: Feature set. Let $F_{M}$ be the set of all features $f$ of the model $M$. Therefore, we have two feature sets considering the context dimensions mentioned above: $F_{\text {location }}=$ $\left\{f_{\text {geographical }}, f_{\text {temporal }}\right\}$ and $F_{\text {user }}=\left\{f_{\text {device }}, f_{\text {activity }}\right\}$.

Definition 2: Value set. Let $V_{f}$ be the set of possible values for a feature $f$. The concrete value of a feature $f \in F_{M}$ at a given point in time is given by $f$.value $\in V_{f}$.

Definition 3: Feature weight. Let $f$.weight $\in[1, \ldots, 5] \subset$ $Q$ be the constant property meaning the importance in the decision process of every feature $f \in F_{M}$, where $f$.weight $=$ 1 means that the feature is definitely not imporant and f.weight $=5$ means that the feature is very important.

Definition 4: Appropriateness factor. Let appr (f.value) $\in$ $[1, \ldots, 5] \subset Q$ be a value for each feature value $f$.value $\in V_{f}$ indicating the appropriateness of a proactive recommendation, where $\operatorname{appr}(f . v a l u e)=1$ means that the recommendation would be not at all appropriate, whereas 5 means that the recommendation would be very appropriate.

Definition 5: Situation model recommendation score. Let $S R S_{M}$ be a value obtained by the combination of the appropriateness factors of feature values and the features' weights of the respective model $M$ for a specific situation.

Based on the previous definitions, we use a model score method based on simple weighting defined as follows:

$$
S R S_{M}=\frac{\left.\sum_{f \in F_{M}} \operatorname{appr}(\text { f.value }) * f . \text { weight }\right)}{w_{M}}
$$

where $w_{M}$ can be seen as a constant, as the weights will be known a priori and will not change during the execution of the recommendation process.

Finally, the recommendation decision score $(S 1)$ can be then calculated by a linear combination of the two respective scores. But to do it, we define an additional parameter called influence needed for the decision process.

Definition 6: Context influence factor. Let $i_{c} \in[0, \ldots, 1] \subset$ $Q$ be the influence of every context dimension belonging to a context model $M$, where their values have to comply with $\sum_{M} i_{c}=1$.

Given that, the global decision score $\mathrm{S} 1$ can be defined as follows:

$$
\begin{aligned}
S 1= & i_{\text {location }} * S R S_{\text {location }}+i_{\text {user }} * S R S_{\text {user }} \\
& \text { with } i_{\text {location }}+i_{\text {user }}=1
\end{aligned}
$$

\section{Evaluation AND Results}

\section{A. Description and objectives}

The aim of this evaluation was to obtain the numerical values of features weights (Definition 3) and appropriateness factors of every feature value (Definition 4) corresponding to the proactivity context modeling associated to the learning domain. Thus the methods proposed above can be applied in the ViSH scenario so as to be able of calculating $S 1$, and as a result, incorporate proactivity to the recommendation process. To achieve it, we asked teachers and scientists (i.e. target ViSH users) to evaluate their perception about the appropriateness of the different feature values and the weight of the features itself involved in the scenario using an online questionnaire.

\section{B. Application to ViSH: features and values}

Before carrying out the evaluation among users, we defined the specific context models used in ViSH focused on determining the location and user context features and their values.

The feature set $F_{\text {location }}$ is composed by the features shown in the first two rows of Table I, where the possible values for them are presented too.

In CARS location is currently one of the most important context parameters, especially in ubiquitous system in which users can access the system from different places and at different moments in time. For that reason, we have divided the location context in two features: temporal and geographical.

For the first one, we considered several periods in a day instead of exactly time values to follow a human being temporal perception.

With regard to the second one, we take into account the difference for teachers between being recommended when they are in or out their common city/working area.

Finally, regarding $F_{\text {user }}$, Table I presents the feature set that is considered in the ViSH scenario to describe the user context together with their possible values.

When analyzing proactivity, user context has proved to be also an important context dimension in terms of "interruptibility" or "time pressure" [12]. These parameters can be derived by combining the activity the user is doing in the current situation analyzed and the device used during that activity.

\section{Demographics and data collection}

Of the 156 people who began the online survey through a publicly available website during the month of February 2013, 104 completed it. $64 \%$ of them were teachers, while $36 \%$ were scientists, being both groups the kind of users for which ViSH is oriented.

The teachers were recruited in schools and high-schools from different countries such as Spain, United Kingdom or Germany, being contacted either directly by e-mail or by disseminating the survey in online teacher groups like the Moodle community. The scientists were recruited from universities like the Universidad Politécnica de Madrid, the University of Cambridge and research centers like the European Schoolnet, the Computer and Automation Research Institute of the Hungarian Academy of Sciences or the Institute for Biocomputation and Physics of Complex Systems.

The gender distribution was $50-50 \%$ as 52 men and 52 women completed the questionnaire, being the age distribution 
TABLE I

APPROPRIATENESS FACTORS OF CONTEXT MODEL FEATURES VALUES.

\begin{tabular}{|c|c|c|c|c|}
\hline Feature & Values & Average & Median & Std. Dev. \\
\hline \multirow{2}{*}{ Geographical } & User is in his city/working area & 4.15 & 4 & 0.87 \\
\hline & User is out of his city/working area & 3.36 & 3 & 1.16 \\
\hline \multirow{4}{*}{ Temporal } & Morning & 3.64 & 4 & 1.12 \\
\hline & Afternoon & 3.45 & 4 & 1.02 \\
\hline & Evening & 3.56 & 4 & 0.95 \\
\hline & Night & 2.65 & 3 & 1.31 \\
\hline \multirow{3}{*}{ Device } & Desktop & 4.20 & 4 & 0.90 \\
\hline & Tablet & 3.69 & 4 & 1.05 \\
\hline & Smartphone & 3.21 & 3 & 1.30 \\
\hline \multirow{10}{*}{ Activity } & Away (user is not in front of the computer/device) & 2.53 & 2 & 1.11 \\
\hline & Idle (user is in front of the computer/device but doing nothing) & 3.06 & 3 & 0.98 \\
\hline & Browsing the platform & 3.98 & 4 & 0.96 \\
\hline & After filling in the profile & 4.05 & 4 & 0.87 \\
\hline & While creating new educational content & 3.52 & 4 & 1.07 \\
\hline & While editing educational content & 3.36 & 4 & 1.12 \\
\hline & While looking for educational content & 4.22 & 4 & 0.85 \\
\hline & After finishing the creation of a new educational content & 3.47 & 4 & 1.07 \\
\hline & While viewing educational content created by others & 3.76 & 4 & 1.06 \\
\hline & After finishing the view of educational content created by others & 3.88 & 4 & 0.99 \\
\hline
\end{tabular}
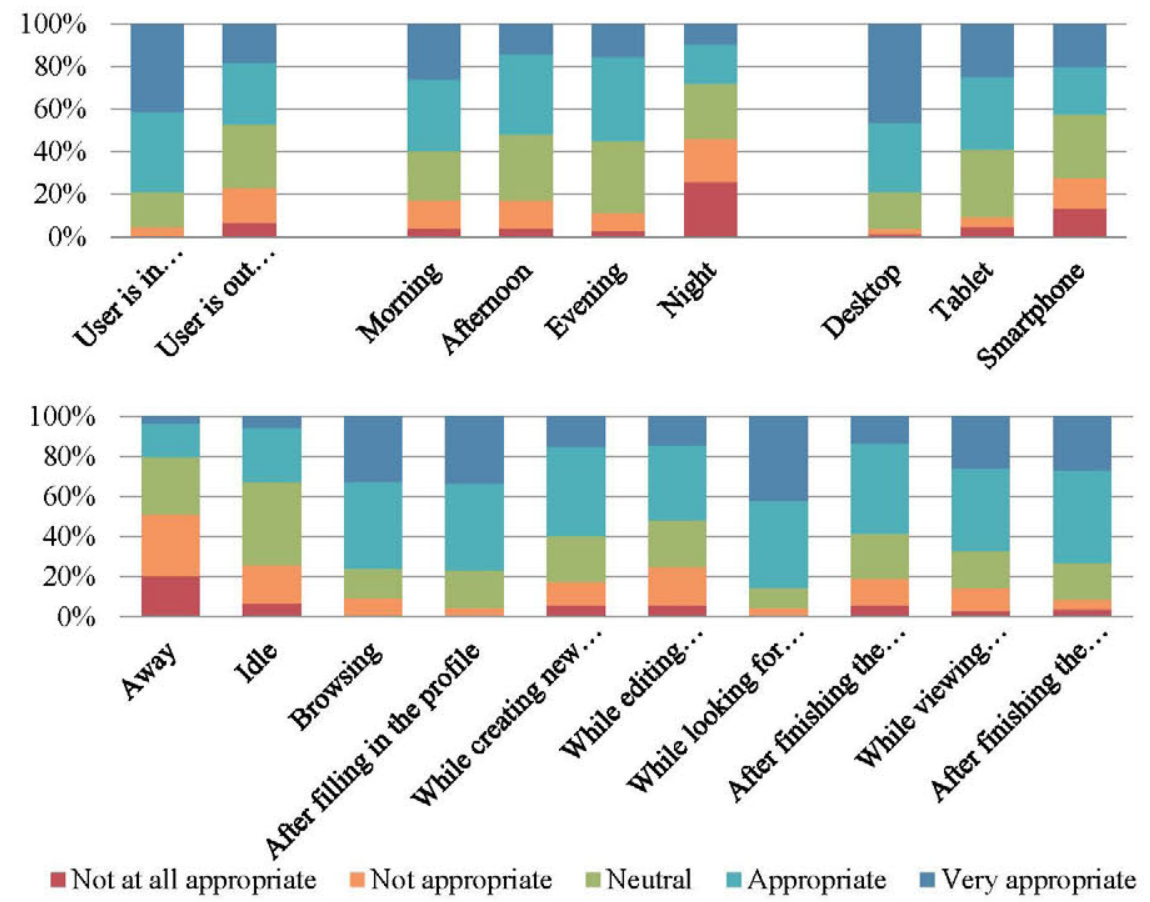

Fig. 1. Evaluation results of appropriateness corresponding to the geographical, temporal, device and activity context feature values.

from 24 to 67 years old, with an average of 39.85 , a median of 39 and a standard deviation of 10.25 .

Concerning the usage frequency of recommender systems in general (e.g. looking up a well rated book or movie) $31.73 \%$ answered "never", 29.81\% answered that they "hardly" use them (i.e. one time per month), $26.92 \%$ answered they use them "regularly" (i.e. at least one time per week) and $11.54 \%$ answered that they use them "frequently" (i.e. almost every day).

Finally, the users were asked about if they had ever heard about proactive recommender systems: $43.27 \%$ answered "yes", whereas $56.73 \%$ said "no".

\section{Results}

Fig. 1 presents graphically the participants' answers to a 5point Likert scale questionnaire when asked about evaluating the appropriateness of being recommended in different contextual situations related to the values corresponding to the geographical, temporal, device and activity features. Table I summarizes the statistical results in terms of appropriateness for every feature value evaluated by the participants.

In the last part of the evaluation, the users were asked to rate the importance of every feature in order to allow us determine their weight in the situation model recommendation score calculation (1). Fig. 7 presents graphically the results provided 


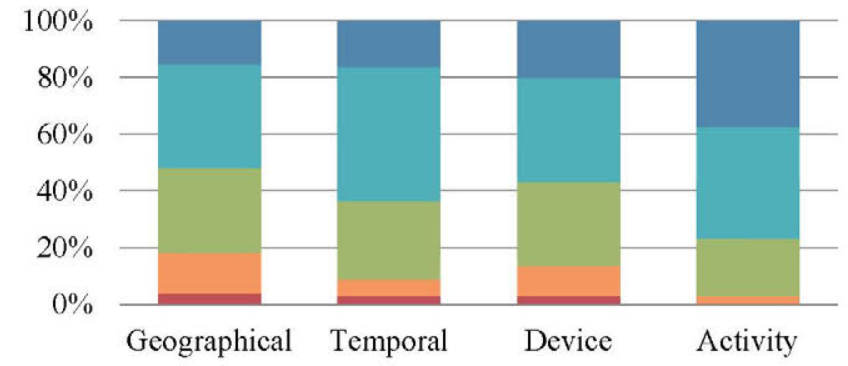

aefinitely not important $\mathbf{m}$ Not important $=$ Neutral $=$ Important $=$ Very important

Fig. 2. Evaluation results of weights corresponding to every context feature.

TABLE II

WEIGHTING OF CONTEXT MODEL FEATURES.

\begin{tabular}{|c|c|c|c|}
\hline Feature weight & Average & Median & Std. Dev. \\
\hline Geographical & 3.45 & 4 & 1.04 \\
Temporal & 3.68 & 4 & 0.92 \\
Device & 3.61 & 4 & 1.02 \\
Activity & 4.12 & 4 & 0.83 \\
\hline
\end{tabular}

by the 5-point Likert scale questionnaire used. Finally, Table 4 shows the statistical results for the features weights.

\section{E. Discussion}

Attending to Nielsen [18], when collecting usability metrics, testing 20 users typically offers a reasonable tight confidence interval. Our sample consisted of 104 participants, so it is appropriate for our quantitative study. As a result of this, we have built a proactivity user modeling valuable for other reseachers that want to include proactive recommendations in their learning scenarios. Additionaly, we have shown that in general the acceptance (i.e. appropriateness) of having this kind of recommendations in TEL is high, as the teachers and scientists evaluated have considered them suitable for their learning processes.

Regarding the appropriateness results illustrated in Fig. 1, it shows two clear situations that are considered inappropriate to be recommended: at night (with an average factor of 2.65) and when the users are away of the computer/device (with an average factor of 2.53). Despite in other scenarios (e.g. tourism) the temporal and activity context to proactively recommend are not totally set, in an educational scenario with teachers and scientist it seems clear that they do not want to be interrupted during their free time for working purposes.

With regard to Fig. 2 it is remarkable that "activity" is clearly the most important feature to take into account when proactively recommending. This outcome is backed by previous results [12] which also shown that understanding the current activity of a user and the level of interruptibility allowed are the most influential parameters in proactivity.

\section{USER INTERFACE FOR PROACTIVE RECOMMENDATIONS}

Our model [3] suggests proactive context-aware recommendations of LOs and similar peers. But when the recommenda- tion is generated, it is sent to the e-learning system to show it in the most adequate way to the user.

In our case we have applied this model in the ViSH scenario. ViSH can be accessed with different kind of devices (i.e. mobile, tablet and desktop/laptop computer) and so the look and feel of the recommendations displayed depends on the device.

Attending to the results achieved in the evaluation process, we implemented in ViSH different user interfaces to incorporate proactive recommendations adapted to every kind of device. Fig 3 illustrates three examples among the different ways the suggestions sent by the recommender engine are presented to the user. Fig 3 a shows a personalized recommendation provided to the user "after finishing the view of educational content created by others". Fig 3b shows a recommendation of both, LOs and similar peers that appears while the user is "browsing the platform". Finally, Fig 3c shows a mobile view in which the user is recommended with a set of suitable resources "after filling in his/her profile".

These interfaces were designed in the participatory design process carried out in the beginning of the project. But after an alpha version period a usability and user experience evaluation was performed. This evaluation consisted on gathering feedback from the first users, clickmaps and scrollmaps [19], property checklists [20] and interviews with potential users [21] to analyze how they used the platform. As a result of this study the interfaces were improved.

\section{CONCLUSION AND FUTURE WORK}

In this article we have studied the appropriateness and importance of providing proactive recommendations to support the learning experience of teachers and scientists involved in a social learning network. To do it we have presented the general definitions and methods needed to implement the situation assessment phase corresponding to the model for generating proactive context-aware recommendations in elearning systems that we proposed in [3]. They allow to calculate the appropriateness of a situation to generate proactive recommendations based on several context dimensions (i.e. location and user context).

To support our approach we have evaluated those methods in a real social learning platform called ViSH related to the GLOBAL excursion European project scenario. Results from the evaluation among educators in the ViSH scenario have leaded us to generate a proactivity user model valuable for other researchers. Furhtermore, we have presented some examples of user interfaces for proactive recommendations implemented in ViSH attending to the outcomes achieved from the evaluation.

Whereas outcomes of this study indicate that perceived appropriateness of receiving proactive recommendations is high, among the future lines of research opened, it would be useful to perform an A/B testing study [22] among ViSH users to evaluate the usability differences between educators using the interfaces proposed and those not using them. Aspects like the usefulness of such type of recommendations in their daily 


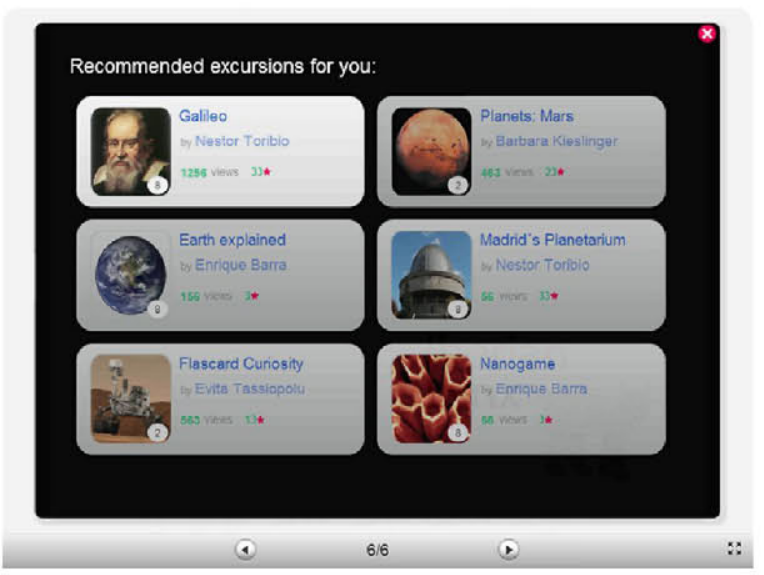

(a)

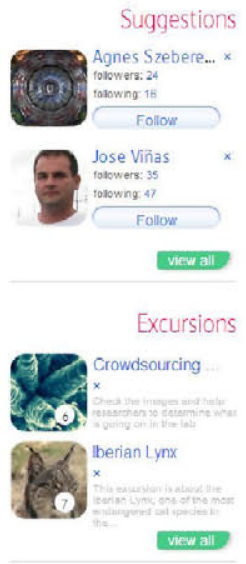

(b)

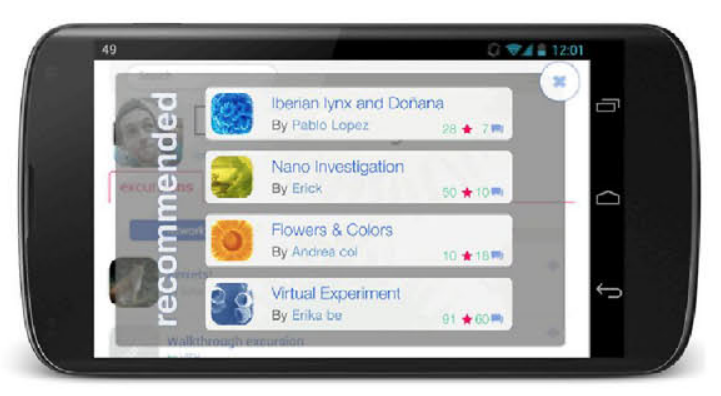

(c)

Fig. 3. Screenshots of ViSH user interfaces for proactive recommendations.

work as teachers, in addition to the influence in the quality of the lessons created thank to the suggestions received could be measured to appreciate the impact of having this kind of recommendation in social learning networks designed for knowledge sharing.

Finally, it would be beneficial for this research to extend the case study to other e-learning platforms so as to apply the methods proposed in other scenarios that want to include proactivity.

\section{ACKNOWLEDGMENTS}

This research work was made possible thanks to funding from the GLOBAL excursion project (European Union under FP7, Infrastructures) and the SAAN project (TIN2010-19138).

\section{REFERENCES}

[1] N. Manouselis, H. Drachsler, R. Vuorikari, H. Hummel, and R. Koper, "Recommender systems in technology enhanced learning," in Recom mender Systems Handbook, F. Ricci, L. Rokach, B. Shapira, and P. B. Kantor, Eds. Springer US, 2011, pp. 387-415.

[2] K. Verbert, N. Manouselis, X. Ochoa, M. Wolpers, H. Drachsler, I. Bosnic, and E. Duval, "Context-aware recommender systems for learning: A survey and future challenges," IEEE Transactions on Learning Technologies, vol. 5, no. 4, pp. 318-335, 2012.

[3] D. Gallego, E. Barra, S. Aguirre, and G. Huecas, "A model for generating proactive context-aware recommendations in e-learning systems," in Frontiers in Education Conference (FIE), 2012, 2012, pp. 1-6.

[4] N. Manouselis, R. Vuorikari, and F. Van Assche, "Collaborative recommendation of e-learning resources: an experimental investigation," Journal of Computer Assisted Learning, vol. 26, no. 4, pp. 227-242, 2010.

[5] M. M. Recker and D. A. Wiley, "A non-authoritative educational metadata ontology for filtering and recommending learning objects," Interactive Learning Environments, vol. 9, no. 3, pp. 255-271, 2001.

[6] K. Verbert, H. Drachsler, N. Manouselis, M. Wolpers, R. Vuorikari, and E. Duval, "Dataset-driven research for improving recommender systems for learning," in Proceedings of the 1st International Conference on Learning Analytics and Knowledge, ser. LAK '11. New York, NY, USA: ACM, 2011, pp. 44-53.

[7] R. Koper, E. Rusman, and P. Sloep, "Effective learning networks," 2005.

[8] E. Garca, C. Romero, S. Ventura, and C. Castro, "An architecture for making recommendations to courseware authors using association rule mining and collaborative filtering," User Modeling and User-Adapted Interaction, vol. 19, no. 1-2, pp. 99-132, 2009.
[9] F. Modritscher, "Towards a recommender strategy for personal learning environments," Procedia Computer Science, vol. 1, no. 2, pp. $2775-$ 2782, 2010.

[10] A. K. Dey, "Understanding and using context," Personal Ubiquitous Comput., vol. 5, no. 1, pp. 4-7, Jan. 2001.

[11] G. Adomavicius and A. Tuzhilin, "Context-aware recommender systems," in Recommender Systems Handbook, F. Ricci, L. Rokach, B. Shapira, and P. B. Kantor, Eds. Springer US, 2011, pp. 217-253.

[12] D. Gallego, W. Woerndl, and G. Huecas, "Evaluating the impact of proactivity in the user experience of a context-aware restaurant recommender for android smartphones," Journal of Systems Architecture, 2013, in press.

[13] A. Ruiz-Iniesta, G. Jimenez-Diaz, and M. Gomez-Albarran, "Recommendation in repositories of learning objects: A proactive approach that exploits diversity and navigation-by-proposing," in Advanced Learning Technologies, 2009. ICALT 2009. Ninth IEEE International Conference on, 2009, pp. 543-545.

[14] European Commission, "Rethinking education: Investing in skills for better socio-economic outcomes," in Communication from the Commission to the European Parliament, the Council, the European Economic and Social Committee and the Committee of the Regions, 2012.

[15] European-Commission, "Supporting the teaching professions for better learning outcomes," in Accompanying the document Communication from the Commission Rethinking Education: Investing in skills for better socio-economic outcomes, 2012.

[16] P. Wastiau, R. Blamire, C. Kearney, V. Quittre, E. Van de Gaer, and C. Monseur, "The use of ict in education: a survey of schools in europe," European Journal of Education, vol. 48, no. 1, pp. 11-27, 2013.

[17] T. Holocher-Ertl, B. Kieslinger, and C. Fabian, "Designing for the users or with the users? a participatory design approach for science teaching in schools," in Proceedings of the 2012 eChallenges Annual Conference, 2012.

[18] J. Nielsen, "Quantitative studies: How many users to test?" in Jakob Nielsens Alertbox, 2006.

[19] K. Choros, "Further tests with click, block, and heat maps applied to website evaluations," in Computational Collective Intelligence. Technologies and Applications, ser. Lecture Notes in Computer Science, P. Jdrzejowicz, N. Nguyen, and K. Hoang, Eds. Springer Berlin Heidelberg, 2011, vol. 6923, pp. 415-424.

[20] P. Jordan, Designing Pleasurable Products: An Introduction to the New Human Factors. Philadelphia, PA, USA: CRC Press., 2000.

[21] P. Desmet, "Measuring emotion: Development and application of an instrument to measure emotional responses to products," in Funology, ser. Human-Computer Interaction Series, M. Blythe, K. Overbeeke, A. Monk, and P. Wright, Eds. Springer Netherlands, 2005, vol. 3, pp. 111-123.

[22] E. Dixon, E. Enos, and S. Brodmerkle, "A/b testing of a webpage," no. US 7975000 B2, 072011. 